\title{
Self-Energy Correction to the Hyperfine Structure Splitting of Hydrogenlike Atoms
}

\author{
H. Persson \\ Gesellschaft für Schwerionenforschung (GSI), Planckstrasse 1, Postfach 110 552, D-64220 Darmstadt, Germany
}

S. M. Schneider and W. Greiner

Institut für Theoretische Physik, Johann Wolfgang Goethe-Universität, Postfach 111 932, D-60054 Frankfurt am Main, Germany

G. Soff

Institut für Theoretische Physik, Technische Universität Dresden, Mommsenstrasse 13, D-01062 Dresden, Germany

\section{Lindgren}

Department of Physics, Chalmers University of Technology and the University of Gothenburg, S-412 96 Göteborg, Sweden

(Received 1 November 1995)

\begin{abstract}
A first testing ground for QED in the combined presence of a strong Coulomb field and a strong magnetic field is provided by the precise measurement of the hyperfine structure splitting of hydrogenlike ${ }^{209} \mathrm{Bi}$. We present a complete calculation of the one-loop self-energy correction to the first-order hyperfine interaction for various nuclear charges. In the low- $Z$ regime we almost perfectly agree with the $Z \alpha$ expansion, but for medium and high $Z$ there is a substantial deviation.
\end{abstract}

PACS numbers: 31.30.Gs, 31.15.Ar, 31.30.Jv

Very recently it was reported that for the first time the hyperfine structure splitting of a hydrogenlike high- $Z$ atom was observed with a high relative accuracy of about $10^{-4}$ at the ESR at GSI, Darmstadt. The transition energy of the ground state hyperfine structure splitting of ${ }^{209} \mathrm{Bi}^{82+}$ was measured to be $\Delta E=5.0840(8) \mathrm{eV}$ [1]. This has challenged theory to perform calculations with comparable accuracy, including also one-loop QED corrections. The new experimental situation opens up a possibility to perform a novel test of QED in a combined strong magnetic field and a strong Coulomb field.

The leading QED effects are of two types: vacuum polarization and self-energy corrections. The vacuum polarization correction is relatively straightforward to compute, using an Uehling-like approximation. This contribution was calculated to be $\Delta E^{\mathrm{VP}}=+0.035 \mathrm{eV}$ quite recently [2]. The remaining Wichmann-Kroll contribution is very small. The one-loop self-energy correction, on the other hand, is more difficult to elaborate, and earlier calculations using an $Z \alpha$ expansion of the Coulomb field are correct only up to order $\alpha(Z \alpha)^{2} m c^{2}$ [3-7]. For heavy elements such an expansion is not reliable. Therefore it is necessary to calculate the self-energy contribution to all orders in $Z \alpha$. In this Letter we present the first complete calculation of this type for different nuclear charges ranging from $Z=1$ to $Z=92$ using similar techniques as published earlier in Refs. [8-12].

First we give a brief outline of the computation of the self-energy correction and later we discuss the numerical results. A more detailed analysis of the calculation of QED corrections to the hyperfine interaction will be presented in a forthcoming paper [13].

In a previous paper it was shown that the unrenormalized self-energy correction for a bound electron state can be written in Feynman gauge as [9]

$$
\begin{aligned}
E_{\text {bou }}(a)= & -\frac{\alpha}{\pi} \sum_{l=0}^{\infty}(2 l+1) \int d k k \sum_{m, q}(-)^{q} \\
& \times \frac{\left\langle a\left|\alpha_{\mu} j_{l}\left(k r_{2}\right) \mathbf{C}_{q}^{[l]}\right| m\right\rangle\left\langle m\left|j_{l}\left(k r_{1}\right) \mathbf{C}_{-q}^{[l]} \alpha^{\mu}\right| a\right\rangle}{E_{a}-E_{m}-\operatorname{sgn}\left(E_{m}\right) k},
\end{aligned}
$$

where $\alpha_{\mu} \alpha^{\mu}=(1-\vec{\alpha} \cdot \vec{\alpha}), C_{q}^{[l]}$ is the $q$ component of the spherical angular tensor of order $l, j_{l}(k r)$ denotes the spherical Bessel function of order $l$, and $|a\rangle$ represents the reference state. There is also a corresponding mass counter term.

To calculate the self-energy corrections to the hyperfine structure we treat the magnetic potential $\vec{A}(\vec{r})$ as a perturbation of the system. This perturbation will affect the binding energy of the bound electron, the wave function, and the bound propagator as already sketched out in Ref. [14]

$$
\begin{aligned}
& E_{a} \rightarrow E_{a}+\langle a|e \vec{\alpha} \cdot \vec{A}(\vec{r})| a\rangle+\cdots, \\
& |a\rangle \rightarrow|a\rangle+\sum_{n \neq a} \frac{\langle n|e \vec{\alpha} \cdot \vec{A}(\vec{r})| a\rangle}{E_{a}-E_{n}}|n\rangle+\cdots,
\end{aligned}
$$

$$
\begin{aligned}
S_{F}\left(\vec{x}_{2}, \vec{x}_{1} ; z\right) \rightarrow & S_{F}\left(\vec{x}_{2}, \vec{x}_{1} ; z\right) \\
& +\int d^{3} x_{3} S_{F}\left(\vec{x}_{2}, \vec{x}_{3} ; z\right) e \vec{\alpha} \cdot \vec{A}\left(\vec{x}_{3}\right) \\
& \times S_{F}\left(\vec{x}_{3}, \vec{x}_{1} ; z\right)+\cdots .
\end{aligned}
$$


Following the diagrammatic depiction of the $S$-matrix formulation, the unrenormalized wave function modification is the nondegenerate part of the diagrams in Figs. 1(a) and 1 (b). The binding energy correction is obtained from the degenerate part after subtracting the corresponding un- linked product of first-order $S$-matrix elements $[15,16]$. The modification of the propagator corresponds to the vertex correction diagram in Fig. 1(c).

The wave-function modification term will take the form

$$
\begin{aligned}
E^{\mathrm{wf}}(a)= & -\frac{\alpha}{\pi} \sum_{l=0}^{\infty}(2 l+1) \int d k k \sum_{q}(-)^{q} \\
& \times \sum_{E_{m} \neq E_{a}} \sum_{n} \frac{\left\langle a\left|e \vec{\alpha} \cdot \vec{A}\left(\vec{x}_{3}\right)\right| m\right\rangle\left\langle m\left|\alpha_{\mu} j_{l}\left(k r_{2}\right) \mathbf{C}_{q}^{[l]}\right| n\right\rangle\left\langle n\left|j_{l}\left(k r_{1}\right) \mathbf{C}_{-q}^{[l]} \alpha^{\mu}\right| a\right\rangle}{\left(E_{a}-E_{m}\right)\left[E_{a}-E_{n}-\operatorname{sgn}\left(E_{n}\right) k\right]},
\end{aligned}
$$

where the sum explicitly excludes $E_{m}=E_{a}$. There is also a corresponding mass counterpart.

The vertex correction leads to the following expression:

$$
\begin{aligned}
E^{\mathrm{vc}}(a)= & -\frac{\alpha}{\pi} \sum_{l=0}^{\infty}(2 l+1) \int d k k \sum_{q}(-)^{q} \\
& \times \sum_{m, n} \frac{\left\langle a\left|\alpha_{\mu} j_{l}\left(k r_{3}\right) \mathbf{C}_{q}^{[l]}\right| m\right\rangle\left\langle m\left|e \vec{\alpha} \cdot \vec{A}\left(\vec{x}_{2}\right)\right| n\right\rangle\left\langle n\left|j_{l}\left(k r_{1}\right) \mathbf{C}_{-q}^{[l]} \alpha^{\mu}\right| a\right\rangle}{\left[E_{a}-E_{m}-\operatorname{sgn}\left(E_{m}\right) k\right]\left[E_{a}-E_{n}-\operatorname{sgn}\left(E_{n}\right) k\right]} F,
\end{aligned}
$$

where we have introduced the function $F$ defined by

$$
F=1+\left[\operatorname{sgn}\left(E_{m}\right)-\operatorname{sgn}\left(E_{n}\right)\right] \frac{k}{E_{m}-E_{n}} .
$$

For the binding energy modification term the formula reads as follows:

$$
\begin{aligned}
E^{\mathrm{be}}(a)= & \frac{\alpha}{\pi} \sum_{l=0}^{\infty}(2 l+1) \int d k k \sum_{q}(-)^{q}\langle a|e \vec{\alpha} \cdot \vec{A}(\vec{x})| a\rangle \\
& \times \sum_{m} \frac{\left\langle a\left|\alpha_{\mu} j_{l}\left(k r_{2}\right) \mathbf{C}_{q}^{[l]}\right| m\right\rangle\left\langle m\left|j_{l}\left(k r_{1}\right) \mathbf{C}_{-q}^{[l]} \alpha^{\mu}\right| a\right\rangle}{\left[E_{a}-E_{m}-\operatorname{sgn}\left(E_{m}\right) k\right]^{2}} .
\end{aligned}
$$

In order to isolate the ultraviolet divergencies we have used the dimensional regularization and generalized the procedure of [17] for an external magnetic interaction. The infrared divergencies can explicitly be shown to cancel. The numerical evaluation of the finite parts is made along the same lines as in $[12,18,19]$.
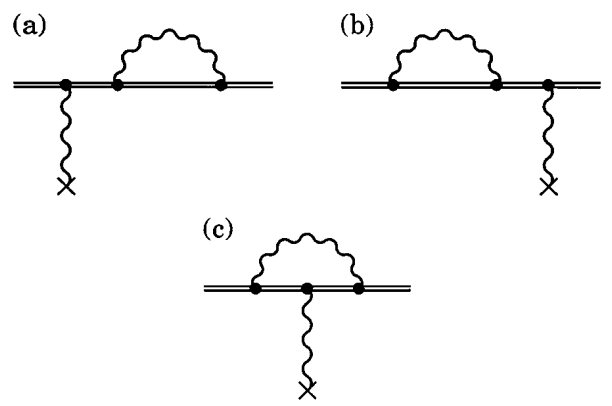

FIG. 1. Graphical representation self-energy correction diagrams. The wave-function modification is shown in (a), (b), and the vertex correction to the magnetic interaction in (c). Double lines indicate wave functions and electron propagators in the Coulomb field. The cross denotes the interaction with the external magnetic field.
To compare our results with the $Z \alpha$ expansion we introduce the function $\tilde{F}$ defined by

$$
\Delta E^{\mathrm{QED}}=\frac{\alpha}{\pi} \Delta E^{1 . \mathrm{ord}} \tilde{F} .
$$

Here, $\Delta E^{1 . o r d}$ denotes the full relativistic first-order energy splitting. Thus the leading relativistic correction is provided by the first-order value and not by $\tilde{F}$. Earlier results for the $Z \alpha$ expansion [3-7] can be summarized as

$$
\begin{gathered}
F(Z \alpha)=\frac{1}{2}-\left[\frac{13}{4}-\ln (2)\right] \pi(Z \alpha) \\
+(Z \alpha)^{2}\left[15.10(29)+\left[\frac{37}{72}-\frac{8}{3} \ln (2)\right] \ln (Z \alpha)\right. \\
\left.-\frac{8}{3} \ln ^{2}(Z \alpha)\right] .
\end{gathered}
$$

It should be noted that the expansion is based on a nonrelativistic point nucleus treatment and $F(Z \alpha)$ is defined as Eq. (9) but with the corresponding nonrelativistic point-nucleus first-order value. The constant term in the expansion is the Schwinger correction of the magnetic moment of the electron.

The values of $\tilde{F}$ and $F(Z \alpha)$ for nuclear charges $Z$, ranging from $Z=1$ to $Z=92$, are given in Table I and are displayed in Fig. 2. For low $Z$ the agreement is perfect, while for higher $Z$ the two results differ substantially. In the numerical calculation a point nucleus charge distribution was used for low $Z$, but for $Z>18$ we have included the effect of a finite nuclear charge distribution both in the self-energy and in the first-order computation.

For the two experimentally interesting cases ${ }^{209} \mathrm{Bi}^{82+}$ and ${ }^{207} \mathrm{~Pb}^{81+}$ we present the theoretical results in Table II. For the ${ }^{209} \mathrm{Bi}^{82+}$ case the self-energy calculation yields $\Delta E^{\mathrm{SE}}=-0.06144(1) \mathrm{eV}$. Combined with the vacuum polarization result of $\Delta E^{\mathrm{VP}}=0.0346 \mathrm{eV}$ the total QED correction amounts to $\Delta E^{\mathrm{QED}}=-0.0268 \mathrm{eV}$. In order to compare to the experimental results one has to add 
TABLE I. The self-energy contribution displayed as $\tilde{F}$ for various nuclear charges $Z$. The numerical results are compared with the values of $F(Z \alpha)$.

\begin{tabular}{rccc}
\hline \hline$Z$ & rms & $\tilde{F}$ & $F(Z \alpha)$ \\
\hline 1 & $\ldots$ & 0.4379 & 0.43805 \\
3 & $\ldots$ & 0.3070 & 0.30783 \\
5 & $\ldots$ & 0.1730 & 0.17634 \\
7 & $\ldots$ & 0.0365 & 0.04681 \\
10 & $\ldots$ & -0.1627 & -0.14026 \\
18 & $\ldots$ & -0.6884 & -0.57762 \\
32 & 4.07 & -1.562 & -1.0716 \\
54 & 4.78 & -2.943 & -1.0656 \\
66 & 5.21 & -3.759 & -0.64854 \\
74 & 5.37 & -4.356 & -0.20921 \\
82 & 5.497 & -5.012 & 0.35762 \\
83 & 5.519 & -5.098 & 0.43736 \\
92 & 5.860 & -5.916 & 1.2430 \\
\hline \hline
\end{tabular}

the first-order hyperfine interaction. The major nuclear effect on this first-order calculation is the extended nuclear charge which can be handled using realistic charge distributions (Fermi and Fermi-Gauss distributions) [21]. The uncertainty between the different nuclear charge distributions is about $0.02 \%$ of the first-order hyperfine interaction. A more limiting uncertainty is given by the extended nuclear magnetization distribution, the BohrWeisskopf effect [22], which has received considerable interest lately [23-26]. We will here use the most elaborated calculation by Tomaselli et al. [25]. This yields a contribution of $\Delta E^{1.0 r d}=5.085(8) \mathrm{eV}$ for the firstorder hyperfine interaction and a total theoretical value of $\Delta E^{1.0 r d}=5.058(8) \mathrm{eV}$. Uncertainties due to missing contributions, like the Wichmann-Kroll magnetic loop correction, are estimated to be rather small.

The magnetic dipole moment of the $\mathrm{Bi}$ nucleus is currently given as $\mu_{\mathrm{Bi}}=4.1106 \mu_{\mathrm{B}}$ [27-29]. This is based on an old NMR measurement in a liquid solution.

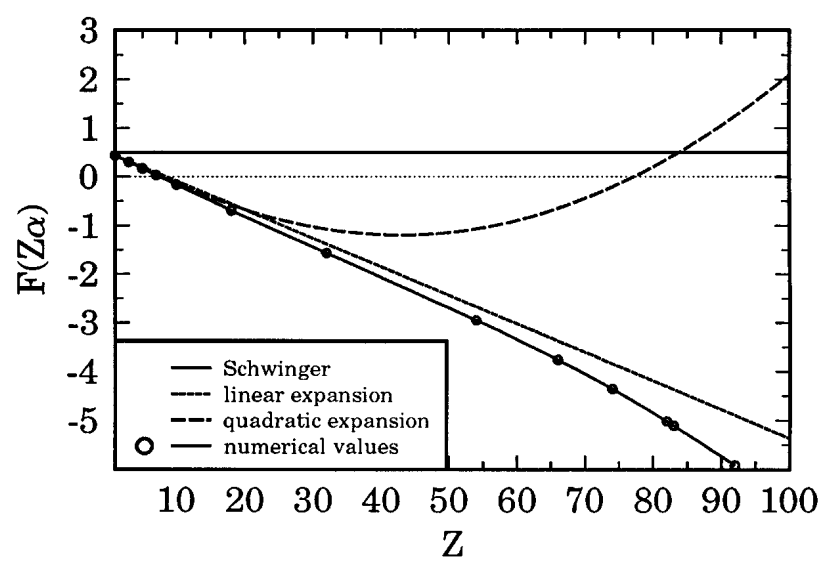

FIG. 2. Graphical comparison of our new numerical values $\tilde{F}$ (full line with circles) and the earlier $Z \alpha$-expansion results. We plotted the Schwinger result (full line), the expansion up to terms proportional to $(Z \alpha)$ (long dashed line), and the expansion up to terms proportional to $(Z \alpha)^{2}$ (short dashed line).

This value includes the diamagnetic correction for a free ion, but not the shift due to the chemical environment. The latter effect is hard to estimate, but it is typically of the order of a few tenths of a percent and in extreme cases it can be even larger [30]. The chemical shift causes presently the largest uncertainty of the theoretical value of the hyperfine interaction. A more precise measurement of the magnetic dipole moment would therefore be highly desirable.

In summary, we have accomplished a complete calculation of the one-loop self-energy correction to the firstorder hyperfine interaction valid for the whole $Z$ range. As required, the agreement with the $Z \alpha$ expansion is almost perfect for low $Z$. For medium and high $Z$ there is a substantial difference resulting from our inclusion of higher-order terms in $Z \alpha$. Also relativistic corrections and finite size effects beyond the first-order value comes

TABLE II. Different contributions to the hyperfine structure splitting of the two experimentally interesting cases ${ }^{207} \mathrm{~Pb}^{81+}$ and ${ }^{209} \mathrm{Bi}^{82+}$. All values are given in $\mathrm{eV}$. The Bohr-Weisskopf modification in the lead case is only estimated in the single particle approach. The error due to the chemical shift on the magnetic moment is not included.

\begin{tabular}{lcc}
\hline \hline & ${ }^{207} \mathrm{~Pb}^{81+}$ & ${ }^{209} \mathrm{Bi}^{82+}$ \\
\hline rms value & $5.497 \mathrm{fm}$ & $5.519 \mathrm{fm}$ \\
Magnetic moment & $\mu=0.592583 \mu_{B}$ & $\mu .1106 \mu_{B}$ \\
First order & $1.2752(2)$ & $5.1917(10)$ \\
Bohr-Weisskopf & -0.034 & $-0.107(7)$ \\
Vacuum polarization (VP) & & 0.0022 \\
$\quad$ Uehling-like loop correction & 0.0062 & 0.0093 \\
$\quad$ Uehling correction of wave function & -0.0001 & 0.0260 \\
$\quad$ Wichmann-Kroll correction of wave function & -0.0148 & -0.0007 \\
Self-energy (SE) & -0.0065 & -0.0614 \\
Sum of QED corrections & 1.2347 & -0.0268 \\
Total & & $5.058(8)$ \\
Experiment & & $5.0840(8)$ \\
\hline \hline
\end{tabular}


in. For the special case of the very accurate measurement of bismuth the theoretical and the experimental values are in fair agreement, keeping in mind the large chemical shift uncertainty.

The authors are very grateful to S. Salomonson, A. Schäfer, W. R. Johnson, P. Mohr, L. N. Labzowsky, and P. Sunnergren for fruitful discussions. The work of H.P. and I. L. was supported by the Alexander von Humboldt Foundation, NFR, and SI. S. M. S. wants to thank the DAAD for financial support from the HSP/II. G. S. acknowledges support by BMBF and by DFG.

[1] I. Klaft, S. Borneis, T. Engel, B. Fricke, R. Grieser, G. Huber, T. Kühl, D. Marx, R. Neumann, S. Schröder, P. Seelig, and L. Völker, Phys. Rev. Lett. 73, 2425 (1994).

[2] S. M. Schneider, W. Greiner, and G. Soff, Phys. Rev. A 50, 118 (1994).

[3] N. M. Kroll and F. Pollock, Phys. Rev. 85, 876 (1952).

[4] R. Karplus and A. Klein, Phys. Rev. 85, 972 (1952).

[5] D. E. Zwanziger, Phys. Rev. 121, 1128 (1961).

[6] S. J. Brodsky and G.W. Ericson, Phys. Rev. 148, 26 (1966).

[7] J. R. Sapirstein, Phys. Rev. Lett. 51, 985 (1983).

[8] S. Salomonson and P. Öster, Phys. Rev. A 40, 5548 (1989).

[9] H. Persson, I. Lindgren, and S. Salomonson, Phys. Scr. T46, 125 (1993).

[10] I. Lindgren, H. Persson, S. Salomonson, and A. Ynnerman, Phys. Rev. A 47, R4555 (1993).
[11] I. Lindgren, H. Persson, S. Salomonson, and L. Labzowsky, Phys. Rev. A 51, 1167 (1995).

[12] A. Mitrushenkov, L. Labzowsky, I. Lindgren, H. Persson, and S. Salomonson, Phys. Lett. A 200, 51 (1995).

[13] S. M. Schneider, H. Persson, G. Soff, W. Greiner, and I. Lindgren (to be published).

[14] P. Indelicato and P. Mohr, Theor. Chim. Acta 80, 207 (1991).

[15] M. Gell-Mann and F. Low, Phys. Rev. 84, 350 (1951).

[16] J. Sucher, Phys. Rev. 107, 1448 (1957).

[17] N. J. Snyderman, Ann. Phys. (N.Y.) 211, 43 (1991).

[18] S. A. Blundell and N. J. Snyderman, Phys. Rev. A 44, R1427 (1991).

[19] K. T. Cheng, W. R. Johnson, and J. Sapirstein, Phys. Rev. A 47, 1817 (1993).

[20] I. Lindgren and J. Morrison, Atomic Many-Body Theory (Springer, Berlin, 1982).

[21] H. de Vries, C. W. de Jager, and C. de Vries, At. Data Nucl. Data Tables 36, 495 (1987).

[22] A. Bohr and V.F. Weisskopf, Phys. Rev. 77, 94 (1950).

[23] M. Finkbeiner, B. Fricke, and T. Kühl, Phys. Lett. A 176, 133 (1993).

[24] S. M. Schneider, J. Schaffner, W. Greiner, and G. Soff, J. Phys. B 26, L581 (1993).

[25] M. Tomaselli, S. M. Schneider, E. Kankeleit, and T. Kühl, Phys. Rev. C 51, 2989 (1995).

[26] L. N. Labzowsky, W. R. Johnson, S. M. Schneider, and G. Soff, Phys. Rev. A 51, 4597 (1995).

[27] Y. Ting and D. Williams, Phys. Rev. 89, 595 (1953).

[28] P. Raghavan, At. Data Nucl. Data Tables 42, 189 (1989).

[29] F. D. Feiock and W. R. Johnson, Phys. Rev. 187, 39 (1969); W. R. Johnson (private communication).

[30] O. Lutz and G. Stricker, Phys. Lett. 35A, 397 (1971). 